\title{
Norois
}

Environnement, aménagement, société

$212 \mid 2009 / 3$

Gestion urbaine et inégalités socio-spatiales France, Brésil

\section{À la recherche d'un coin de paradis ? Paroles habitantes au Clube de Campo de Alvorada Aldeia (Camaragibe - PE, Brésil)}

Looking for a piece of paradise? Dwellers' words within Clube de Campo de Alvorada, Aldeia (camaragibe - PE, Brazil)

\section{Fanny Vuaillat}

\section{(2) OpenEdition}

Journals

\section{Édition électronique}

URL : https://journals.openedition.org/norois/2923

DOI : $10.4000 /$ norois.2923

ISBN : 978-2-7535-1560-4

ISSN : $1760-8546$

\section{Éditeur}

Presses universitaires de Rennes

Édition imprimée

Date de publication : 1 décembre 2009

Pagination : 41-52

ISBN : 978-2-7535-1034-0

ISSN : 0029-182X

\section{Référence électronique}

Fanny Vuaillat, "À la recherche d'un coin de paradis ? Paroles habitantes au Clube de Campo de Alvorada Aldeia (Camaragibe - PE, Brésil) », Norois [En ligne], 212 | 2009/3, mis en ligne le 01 décembre 2011, consulté le 13 janvier 2022. URL : http://journals.openedition.org/norois/2923 ; DOI : https://doi.org/10.4000/norois.2923 


\title{
À LA RECHERCHE D'Un COIN DE PARADIS ? \\ Paroles habitantes au Clube de Campo de Alvorada \\ Aldeia (Camaragibe - PE, Brésil)
}

\author{
FANNy VUaillat \\ CESTAN - ESO UMR 6590 CNRS \\ (Université de Nantes), \\ Chemin de la Censive-du-Tertre, BP 81227 - 44312 Nantes cedex 3, France \\ fanny.vuaillat@univ-nantes.fr
}

\begin{abstract}
RÉSUMÉ
Le Clube de Campo Alvorada est un club de fin de semaine datant des années 60 où depuis quelques temps, des habitants de Recife viennent s'installer à l'année. Une dizaine d'entretiens semi-directifs ont été réalisés auprès de ces nowveaux habitants afin de faire émerger les figures de cette nowvelle manière d'habiter. L'image d'un lieu rêvé ou d'un coin de paradis est omniprésente dans les discours et pour protéger cet endroit, l'organisation, la discipline et la sécurité sont essentielles. En outre, si l'homogénéité sociale est primordiale pour préserver la tranquillité du lieu, les membres du club semblent former une communauté d'intérêts plus qu'une communauté affinitaire. Aussi, l'échelle résidentielle apparaît-elle comme maitrisable par les habitants bien qu'ils se plaignent fortement des modalités de gestion. Enfin, il existe parfois un sérieux décalage entre le rêve d'habiter et les déceptions du quotidien. Les discours habitants sont aussi faits d'une désillusion qui apparâ̂t au fil du temps.
\end{abstract}

MoTS CLÉ : discours habitant-copropriété-fermeture résidentielle-club de campagne - Recife-Brésil

\footnotetext{
ABSTRACT

Looking for a piece of paradise? Dwellers' words within Clube de Campo de Alvorada, Aldeia (camaragibe - PE, Brazil)

Clube de Campo Alvorada was a week-end country club when it originally opened back in the Sixties. Inhabitants of Recife have been progressively taking residence in this place over the recent years. Around ten in-depth interviews were carried out with new users in order to identify what is motivating the seek for this new way of residency. The idea of a dreaming place, like a kind of paradise is redundantly omnipresent. Organisation, order and security are essential to protect this paradise. Although social homogeneity is equally paramount in order to keep the place peaceful, the members express more a community of interests than a community of affinity. Moreover, the residential scale is primarily the scale of the space which the inhabitants can keep control of, although they might complain the management modes. Lastly, there is sometimes a gap between the dream of living there and the disappointments of the day to day routine. The inhabitants' words are also made of this disillusion that appears as time goes.
}

KEY WORDS : dwellers' words - collective ownership - gated community - Country Club - Recife-Brazil 
À moins de vingt kilomètres de Recife (Pernambouc; Brésil), Aldeia, point le plus haut de la commune de Camaragibe, s'étend le long d'une route au milieu de la mata atlântica verdoyante (forêt tropicale du littoral atlantique) et des anciens engenhos. Ces derniers sont des propriétés foncières destinées à la fabrication du sucre. Ils comprennent la plantation, les locaux de production, la résidence du propriétaire (Casa Grande) et les anciennes habitations des esclaves (Senzala). Dès les années 1960, les premiers clubs de fin de semaine se construisent sur ce plateau pour offrir aux classes aisées de Recife un lieu récréatif, aéré et sélectif, alternative séduisante aux plages surpeuplées du littoral nordestin.

Le Clube de Campo Alvorada est un country club comme il s'en est développé de nombreux aux périphéries de diverses villes du monde. Ce type d'habitat renvoie à la diversité des formes d'auto-enfermement résidentielles, phénomène de mieux en mieux connu grâce à la structuration en réseau ${ }^{1}$ des chercheurs travaillant cette question et à la récente publication d'articles ou d'ouvrages (Caldeira, 1996; Billard et al., 2005; Glasze et al., 2005 ; Capron, 2006 ; Degoutin, 2006). Les cinq continents ont fait l'objet de recherches qui ont révélé la globalité du phénomène, malgré l'observation d'une diversité morphologique ou typologique importante.

Au sein de ces divers travaux, l'Amérique latine a très vite dévoilé une réalité ancienne et complexe en matière de fermeture et de sécurisation résidentielle. Ce continent urbain et inégalitaire connaît une explosion de ce type d'habitat et le développement des countries clubs participe grandement à ce phénomène, comme le montrent les analyses les concernant dans l'arrière pays de Buenos Aires (Thuillier, 2001 ; Lacarrieu et Thuillier, 2004 ; Capron, 2006). Dans le cas brésilien, nous nous sommes intéressés au Clube de Campo Alvorada, situé au sein de la Région Métropolitaine de Recife, capitale la plus inégalitaire du pays le plus inégalitaire du continent et du monde. Les inégalités socio-spatiales et la ségrégation ne sont pas nouvelles au Brésil, encore moins dans l'État du Pernambouc. Les structures sociales et spatiales de la société esclavagiste et patriarcale (Freyre, 1933, 1936) transparaissent encore dans les formes urbaines contemporaines (Leitão, 2005). Les formes de la fermeture et de la sécurisation des espaces résidentiels sont nombreuses et quasiment omniprésentes au sein de l'habitat dit formel (à opposer à l'habitat dit informel qui constitue la majorité de l'habitat des populations les plus pauvres). Les espaces résidentiels fermés sont majoritairement des condominios fechados et se divisent en deux grandes catégories : les condominios verticaux que constituent les nombreuses tours qui s'érigent au cœur de Recife et les condominios horizontaux qui regroupent les ensembles fermés de maisons individuelles ou de petits collectifs. Les countries club, dont nous analysons ici un exemple, sont des condominios horizontaux et représentent une des formes de la fermeture résidentielle au sein du vaste panel typologique présent à Recife. L'analyse du développement des countries clubs a un intérêt majeur dans le sens où elle permet de questionner la banalisation de cette forme d'habiter se basant sur les principes sélectifs du club dans la construction des espaces urbains contemporains.

Parmi l'ensemble des recherches réalisées sur les espaces résidentiels fermés et sécurisés, le constat d'un manque de connaissance des parcours, des motivations et des représentations des habitants a été souligné à plusieurs reprises. Plusieurs travaux ont toutefois déjà initié cette approche (Capron, 2006; Vuaillat, Madoré 2008; Le Guirriec, 2008), notamment en privilégiant une démarche discursive, au cours de laquelle les habitants dévoilent leurs pratiques, leurs désirs, leurs craintes, leurs exigences ou leurs déceptions. Le recueil de la parole habitante est donc incontournable pour appréhender en profondeur les ressorts du phénomène et mettre en lumière les rapports que les habitants tissent avec leur espace résidentiel, leur ville ou avec les Autres. Au total, tenter de saisir ce phénomène par une contextualisation des résidences étudiées et par le discours des habitants permet d'approfondir la connaissance portant sur la diversité des figures possibles, tant sur la forme des résidences que sur les modes de gestion, les types de populations et in fine les impacts sur les espaces urbains. 
Pour atteindre cet objectif, nous nous sommes entretenus avec dix habitants permanents du club au moyen d'entretiens semi-directifs, d'une durée comprise entre vingt et quarante minutes chacun. Les quelques questions posées aux résidants étaient volontairement larges dans le but de laisser libre cours au discours d'existence, de vie et aux récits des expériences personnelles. La grande richesse des informations récoltées n'exclut pas certaines limites, qu'il est important de signaler afin d'empêcher tout malentendu quant à la généralisation des conclusions. Cette méthode vise à recueillir le discours d'existence de l'habitant afin d'en faire émerger les redondances communes et les contradictions repérées dans l'ensemble des discours. C'est à partir de ce matériau, fait d'images récurrentes et de paradoxes, que s'exprime un mode d'habiter particulier. Evidemment, l'effet de contexte est important et relativise fortement les conclusions qui demeurent exclusives à l'espace et aux populations étudiés, interdisant ainsi toute généralisation abusive.

Les entretiens présentés ici font partie d'une étude plus vaste sur la question de l'enfermement résidentiel au Brésil et en France. Au Clube de Campo Alvorada, nous nous sommes entretenus avec trois hommes et sept femmes, âgés de 40 à 70 ans (l'âge moyen étant de 55 ans), ce qui représente $10 \%$ des ménages habitant à l'année au Clube de Campo. Leurs revenus varient entre R\$ 2500 (l'unité monétaire brésilienne est le réal, R\$ en abréviation) et R\$ 20 000, pour une moyenne de R 8350 (environ 3000 euros en 2008), soit plus de vingt fois le salaire minimum brésilien, confirmant leur appartenance aux classes aisées. La moitié des habitants rencontrés est mariée, l'autre moitié étant composée de célibataires, de veufs ou de divorcés et seulement deux femmes ont des enfants (entre 14 et 17 ans) vivant dans le club. En revanche, la quasi-totalité des personnes avec lesquelles nous nous sommes entretenus disent recevoir régulièrement enfants, petits-enfants ou neveux au club. Nous terminerons en rappelant les difficultés à enquêter dans ce type de résidence fermée avec contrôle des accès : outre la barrière de la langue (les entretiens ont bien évidemment été réalisés dans la langue maternelle des enquêtés, c'est-à-dire en portugais), cette entreprise nécessite du temps et de la patience pour gagner la confiance des résidants, d'autant plus que nombre d'entre eux sont venus résider dans ce country club justement dans un souci de protection (cf. infra). Comme souvent, ce climat de confiance, indispensable à la réalisation du travail d'enquête, a été obtenu grâce à l'activation d'un réseau de connaissances que nous avons dû constituer au préalable.

Après une présentation du club étudié, nous verrons les différentes figures mises en évidence par l'analyse des discours habitant recueillis. Nous montrerons que dans ce lieu de vie particulièrement idéalisé, la vie communautaire, fondée sur l'intérêt plutôt que sur l'affinité, suscite parfois des conflits et des déceptions au fil du temps.

\section{Le Clube de Campo Alvorada}

Aldeia, à l'ouest de la municipalité de Recife, est un espace valorisé par la bourgeoisie recifense. Dès le $\mathrm{XvI}^{\mathrm{e}}$ siècle, cette région a connu un fort développement de l'agro-industrie sucrière avec la construction de nombreux engenhos, maintenus jusqu'au milieu du $\mathrm{xx}^{\mathrm{e}}$ siècle. À cette époque, une communauté étrangère, nord-américaine notamment, était très présente, ce qui a renforcé la valorisation du site aux yeux des classes aisées pernambucanaises.

En 1962, deux premiers clubs se construisent sur les sites de deux anciens engenhos et accueillent pour les week-ends et les vacances des familles de Recife. Ce sont le Sete Casuarinas (trente hectares) et le Clube de Campo Alvorada, étudié ici (25 hectares). Puis, en 1978, sur le territoire d'Aldeia, un couple d'Américains, John et Lígia Fryer, crée une école internationale pour assurer l'éducation de leurs enfants et plus généralement de la communauté. La création de cette école privée et internationale, l'Escola Internacional de Aldeia, et ses activités de lobbying sera le point de départ d'investissements publics importants pour la construction d'une route et pour l'électrification du plateau. C'est à partir de cette époque que plusieurs membres de ces deux premiers clubs viennent s'installer à l'année profitant de l'installation d'infrastructures publiques 
de base, le club se chargeant du reste, à savoir l'adduction d'eau potable, la collecte des déchets, mais surtout l'installation d'équipements de loisirs.

Dès le début des années 1980, d'autres résidences fermées se construisent sous la forme de club ou de copropriété, la loi structurant les condomínios (contrat de copropriété en vigueur au Brésil) n'existant pas encore, amplifiant ainsi l'installation d'« urbains » à Aldeia. Mais, l'explosion n'apparaît qu'à la fin des années 1990 et se poursuit jusqu'à aujourd'hui avec la construction de quinze condomínios fechados (copropriétés fermées) entre 1998 et 2006. Aujourd'hui, les mutations des modes de vie, la démocratisation de l'automobile et l'amélioration des infrastructures permettent à une partie de la population de la capitale du Pernambouc de venir s'installer, au vert et au frais, sur ces hauteurs. Selon la mairie de Camaragibe, environ 4000 personnes résident à l'année dans les condomínios fechados d'Aldeia. Ce chiffre est sous-estimé car il ne prend en compte que les ensembles résidentiels structurés en condominio, oubliant ainsi les clubs et les conjuntos (ensembles résidentiels n'ayant pas signé de contrat de copropriété formel, forme relativement courante au Brésil).

Le Clube de campo Alvorada, un des plus anciens sur ce plateau, compte 251 membres, soit 251 parcelles, 202 maisons construites, dont 50 sont déclarées en résidences principales. Le terrain du club est entièrement ceint par une clôture barbelée d'environ un mètre quatre-vingt, cachée par une haie végétale. L’entrée est rendue imposante par la présence d'une guérite, d'une double voie d'entrée (pour membres et pour visiteurs) et d'une voie de sortie (Photo 1). Le contrôle des accès s'effectue par des gardiens, postés dans cette guérite. Un interphone permet de s'identifier. Les gardiens prennent alors contact avec la personne visitée afin d'autoriser ou non l'entrée du visiteur. Des caméras de vidéosurveillance enregistrent le numéro d’immatriculation de chaque véhicule. Enfin, même si les membres du club ont une voie d'accès privilégiée, la barrière ne s'ouvre que s'ils ont été formellement reconnus par les gardiens en poste.

Une route fait le tour du club et dessert les impasses non bitumées donnant accès aux maisons (Figure 1). Pour déambuler dans le club et atteindre les équipements de loisirs, divers sentiers parcourent la forêt. Les aménités et les équipements collectifs sont multiples : piscine, courts de tennis, terrain de football, de basketball, salle de gymnastique, bibliothèque, salle de projection et de conférence, sauna, salle de jeux, salle de billard, salle des fêtes, restaurant, chapelle, etc. La gestion du club est assurée par un président, élu par les chefs de famille. Ensuite, il existe un directeur par thème (par exemple : comptabilité, loisirs et fêtes ou encore entretien). Le club

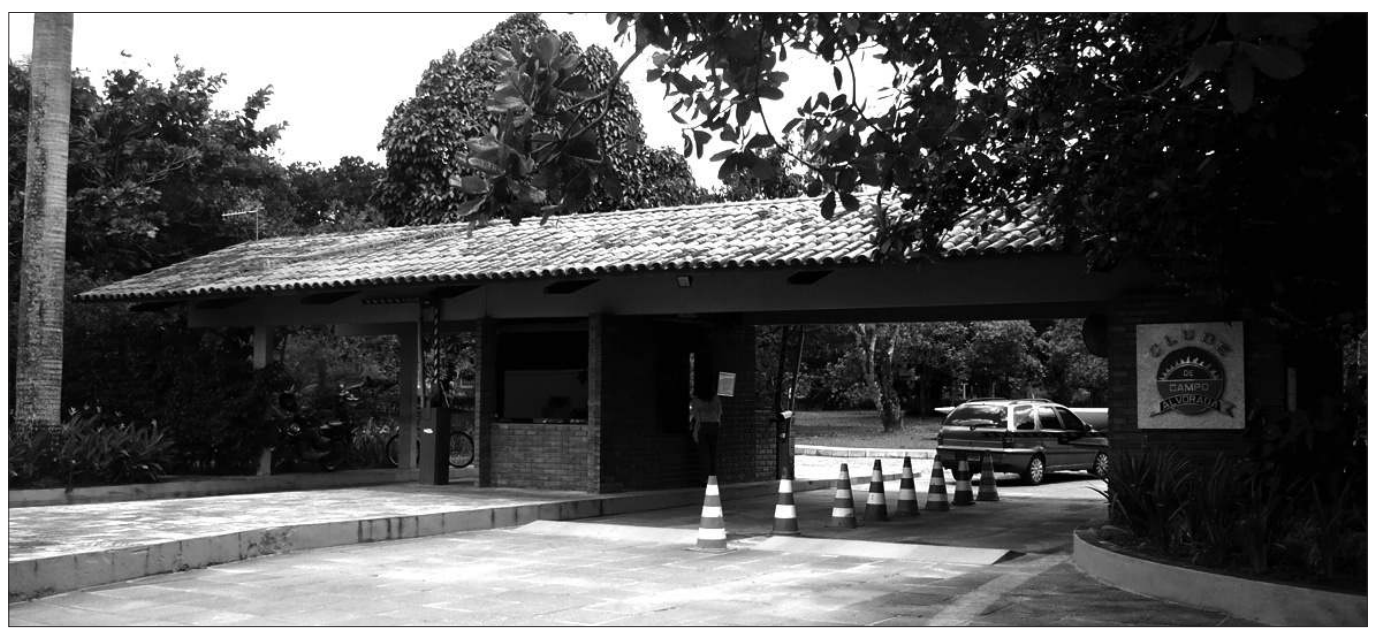

Photo 1 : L'entrée du Clube de Campo Alvorada The gate of Clube de Campo Alvorada 
emploie trente-neuf salariés, dont vingt-deux pour la sécurité, les dix-sept autres étant chargés des tâches administratives, de l'animation et de l'entretien. Pour financer cette gestion, les charges mensuelles par membre s'élèvent au montant du salaire minimum, soit environ R\$ 420 en 2008 (150 euros).

Enfin, pour être membre du club, il faut s'acquitter d'un titre d'une valeur de R\$36000 (environ 12500 euros, fin 2008), qui ouvre le droit de construire une maison sur un terrain - lorsqu'un membre décide de vendre sa maison, il doit vendre aussi le titre de club - et offre l'accès total au club et à tous ses équipements. Il ne peut y avoir plus de membres que de parcelles disponibles. En revanche, tous les membres n'ont pas souhaité utiliser leur parcelle pour construire. Par ailleurs, il ne suffit pas de payer, il faut aussi être autorisé à acheter un titre. Pour cela, chaque nouvel acquéreur potentiel doit être coopté par deux membres. Une fiche le décrivant est affichée pendant un mois dans le club. Chaque membre peut accoler un avis défavorable qui rendra alors impossible l'adhésion au club du prétendant. Ces conditions d'accès, particulièrement exigeantes, inhérentes aux clubs, visent à garantir un certain entre-soi, nous y reviendrons par l'analyse du discours habitant.

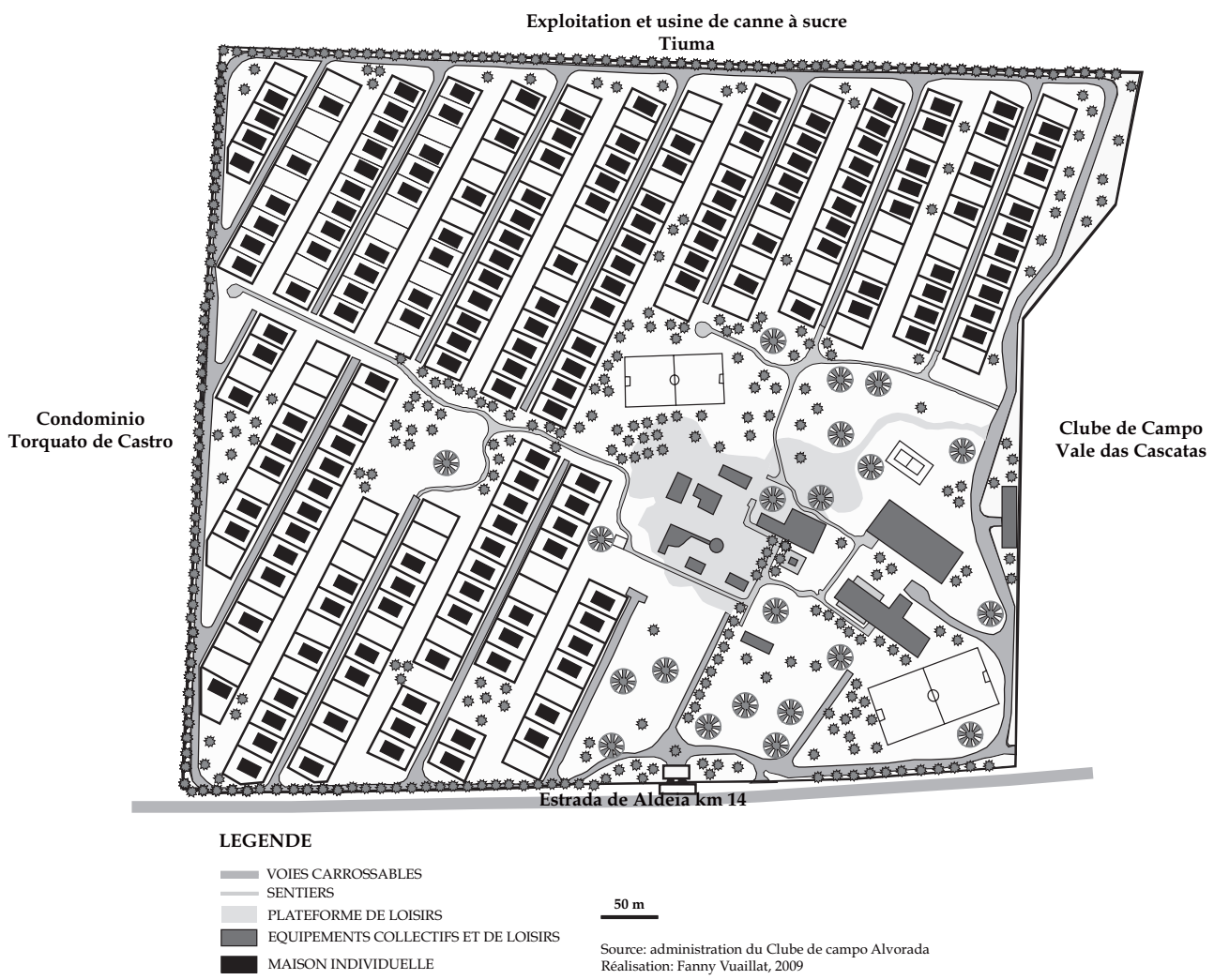

Figure 1 : Plan du Clube de Campo Alvorada Clube de Campo Alvorada's map 


\section{À la recherche du paradis perdu}

À l'entrée du bâtiment qui abrite le secrétariat du club et la bibliothèque, est accrochée une petite céramique peinte sur laquelle est écrit : "Là fora, a chwva, o vento, o frio. Aqui, amor, paz e alegria » («Là-bas, dehors, la pluie, le vent, le froid. Ici, l'amour, la paix et la joie. »). Ce petit écriteau est tout à fait représentatif et significatif de l'ensemble de la communication du club sur cette image d'ilot de paradis au milieu du chaos qui règne à l'extérieur. Le club serait un pays de cocagne où la végétation, les animaux, la Nature en somme y serait libre et luxuriante pour le bien de ses membres. Il est vrai que deux zones de protection naturelle existent dans les documents d'aménagement et de planification collectifs. Ce zonage municipal soumet donc le Clube de Campo Alvorada à ces deux législations (ZCA - Zone de protection environnemental et ZPUR - Zone de protection à urbanisation restreinte). Le rapport entre espace bâti et végétation est ainsi fortement réglementé, laissant une grande partie de l'espace à la forêt.

Dans les discours, les habitants ont beaucoup évoqué la qualité paysagère et naturelle du site. Ils discourent largement sur la beauté de la nature et l'associent directement au calme, à la paix et à la tranquillité. Ces qualificatifs sont récurrents et justifient en grande partie le plaisir d'habiter. Mais, au-delà d'une simple appréciation du caractère agréable du club, les habitants ont fait état du bonheur d'avoir trouvé le paradis, l'agrément du club est aussi synonyme, de leurs points de vue, de liberté. Le sentiment d'être libre est récurrent dans le discours recueilli : libre de ses journées, libre de ses activités, etc.

L’impression de paradis perdu, enfin trouvé ou retrouvé, est amplifié par le fait que les parents voient dans le club le lieu idéal pour élever leurs enfants, soit en se rappelant la liberté de leur propre enfance, soit pour pouvoir leur offrir un cadre de vie agréable, des loisirs et un espace sécurisé. La présence d'aménités aussi participe grandement du rêve d'habiter " au paradis », dans le sens où tous ces aménagements appellent au plaisir, à la détente et au divertissement. Evidement, le fait que ce soit un club, les structures mises en place restent des structures de vacances ou du moins de week-end. Les résidants permanents vivent donc au sein d'un espace de loisirs, ce qui participe au rêve d'habiter dans la mesure où le mythe des vacances toute l'année est mis en scène.

\section{Extraits d'entretien}

«L'air est très doux, super agréable. On a la meilleure température ici à Aldeia, le temps est toujours très agréable. On avait vraiment le souci du vert, de maintenir nos maisons valorisées, il y a peu de pollution. »

«Ce que j'aime, c'est ça... le silence. C'est ce vent, cette odeur, sans pollution. Si elle vient jusque là, il en vient que très peu, on ne sent presque rien. Et puis, cette tranquillité. »

"Ce que j'aime ici, Ave Maria! C'est le calme, c'est apaisant. Vous pouvez sortir vous divertir, rester ici avec des amis, je trouve que c'est super tout est bien."

"Ce que j'aime le plus c'est la nature, les plantes, les oiseaux, les petits singes, il en vient plus de 40 par jour. »

"Ce sont les animaux que j'aime, les plantes, le silence, cet air que l'on respire. Ça me fascine. »

"Je me sens comme au paradis, je suis au paradis sans avoir besoin de mourir."

"Quand je suis arrivée ici, c'était un oasis, je venais de Recife, ici à Aldeia, je me sens mieux, je me sens plus en sécurité. »

"Je suis amoureuse de cet endroit. Moi et mon mari."

"Je trouve qu'ici c'est vraiment un paradis. Boa Viagem (quartier résidentiel aisé de front de mer à Recife), c'est bien, mais je préfère ici. Je ne sais pas la campagne... j’aime la campagne! »

"Ici c'est un don de Dieu. Parce que quand on veut sortir, jouer, tout, on sort, mais quand on veut du calme, on reste ici. C'est très bien ici, on n'a jamais pensé à partir, on a fait un sondage à la maison et personne ne veut descendre en ville. » 
"C'est très confortable. Je trouve qu'ici on travaille mieux, loin de la violence, loin de la pollution. Je vais même dire que l'on a une qualité de vie privilégiée. Je me sens très bien, très sereine. »

"J'ai acheté ici pour venir les week-ends, mais j'ai tellement aimé, c'est un paradis, alors je suis venue vivre. Ça fait 20 ans. »

"Je trouve le lieu merveilleux, il y a la sécurité, l'eau."

"C'est super pour les enfants parce que le club offre beaucoup de choses. En plus de la sécurité, il y a tous ces loisirs. Il y a un sauna, une salle de gym, il y a des fêtes. C'est super de vivre ici, c'est très bien, j'aime beancoup. »

"Pour moi c'était mieux d'élever mes enfants ici. J'habitais à Olinda (dans la banlieue de Recife) et ils vivaient prisonniers. Ici c'est très bien pour élever des enfants, j'ai beaucoup aimé. »

"J'aimais voir mes enfants libres, c'était super, ils aimaient beaucoup. »

\section{Organisation, discipline et sécurité : protéger son paradis}

Les qualités du club sont la plupart du temps évoquées en comparaison avec l'extérieur, le dehors, la ville. En effet, la totalité des habitants avec lesquels nous nous sommes entretenues sont d'anciens habitants de Recife ou d'Olinda - sa ville mitoyenne - et discourent sur les avantages du club en décrivant la ville comme un lieu bruyant, sale et dangereux qu'il a fallu fuir. Moins loin, l'extérieur, c'est aussi les espaces mitoyens du club, l'usine proche, la rue devant le club ou encore les autres condomínios construits plus récemment. Aux yeux des habitants, le club est et se doit d'être le contraire de ce qui se passe à l'extérieur. L'organisation, la discipline des employés et des membres du club, ainsi que la sécurité sont des éléments essentiels afin de protéger ce "petit coin » de paradis du chaos extérieur.

La sécurité n'est pas évoquée en premier lieu pour décrire les qualités du club, mais cette notion devient redondante dès lors que les habitants décrivent les facilités quotidiennes à vivre à Alvorada. À la différence de la vie qu'ils ont pu vivre à Recife, les habitants ne se préoccupent plus ou très peu des questions de sécurité, s'en remettant totalement aux équipements et aux équipes de surveillance du club. Les habitants semblent donc être délivrés de cette contrainte. À l'intérieur du club, il n'existe aucun mur, aucune barrière, toutes les parcelles sont ouvertes et les habitants laissent leur maison ouverte sans se soucier d'une éventuelle intrusion, ce qui participe grandement au sentiment de liberté qu'ils décrivent.

Selon les discours, ce paradis doit être protégé de la « décadence » et de la "pagaille » extérieure. Sur ce thème, la référence à la favela est fréquente, soit pour décrire les Autres, l'extérieur, soit a contrario pour décrire ce que n'est pas et ne doit pas être le club. Cette observation confirme les résultats d'autres travaux, notamment sur les countries de Buenos Aires. La qualité de vie, le rapprochement avec la nature, le rejet des maux de la ville s'accompagnent de la quête d'un espace aseptisé dans lequel un règlement intérieur drastique s'applique à produire un « espace sécurisé, calme et purifié, éliminant tout risque » (Lacarrieu, Thuilier, 2004, p. 162). Ainsi, l'organisation et la bonne gestion du club sont indispensables et appréciées, la discipline et la confiance des équipes de surveillance apparaissent comme essentielles. Beaucoup d'employés travaillent depuis longtemps à Alvorada, ce qui est rassurant pour les résidants qui leur accordent leur confiance. Enfin, les dispositifs de surveillance sont appréciés et jugés nécessaires, à savoir les barbelés, la guérite et le poste d'identification afin d'éviter toutes intrusions, invasions ou dégradations. En ce sens, au cours de l'hiver 2008 a été voté le renforcement des dispositifs de sécurité du club, sans qu'il n'ait été fait état d'une intrusion justifiant cette décision. Il a été décidé de surélever la guérite à l'entrée du club de façon à protéger les gardiens d'une éventuelle attaque, pour leur permettre de prévenir secours et policiers. Le système central de vidéosurveillance va être également modernisé et étendu à l'ensemble du club. Les arguments annoncés relèvent de la nécessité de prévenir tout problème potentiel. La surenchère sécuritaire est à l'œuvre sans que des éléments concrets puissent la justifier. 


\section{Extraits d'entretien}

"La tranquillité, de ne pas être ennuyé par ces choses urbaines, personne qui peut frapper à ta porte. »

"Il n'y a pas de perturbations, pas de mendiants à la porte. "

"Ce n'est pas une favela ici, non?»

«Ah! J'aime cette tranquillité, ce silence, c'est exactement le contraire de ce que j'avais en centre ville. Là bas, la rue était bruyante, tout était compliqué. Là bas, on n'est jamais sûr quand tu sors dans la rue.»

"J'aime beancoup ici, j'ai peur de marcher en ville. Quand je vais chez ma sœur, chaque fois que j'arrive en voiture, je regarde partout, de tous les côtés, j'ai peur. Ici, non, je ne me préoccupe pas de ça. »

"J'allais beaucoup à la plage quand j'étais jeune, mais aujourd'hui nos plages sont polluées, ce ne sont pas les plages d'avant. Parfois, il y a des petites bêtes, des cafards dans les rochers, alors je n'aime pas trop, je ne vais plus à la plage, je reste ici. »

"Je trouve qu'il est quasiment impossible de vivre dans nos villes aujourd'hui, principalement pour cette question de violence. »

"Nous avons un service de surveillance le jour et un la nuit. Nous habitons ici depuis 11 ans et il ne s'est jamais rien passé ici à l'intérieur, toujours à l'extérieur mais jamais à l'intérieur. "

"C'est très bien, très bien organisé, l'organisation est très bien. "

"C'est nécessaire, ici à Aldeia, c'est nécessaire de contrôler parce que sinon tout le monde entrerait et ici ce ne serait plus privé, ce serait... »

"On a besoin d'un système de surveillance pour que des gens de l'extérieur ne soient pas tentés d'envahir le club.»

"Ce sont d'anciens employés, depuis 10,11,18 ans, Aldo il travaille depuis 18 ans ici, c'est celui du matin. J'aime bien ça, je trouve que ça apporte une sécurité. »

\section{Une communauté... d'intérêts?}

Au sein des travaux effectués sur le sujet de l'auto-enfermement résidentiel, les hypothèses et les affirmations d'une quête d'entre-soi ont été nombreuses (Pinçon et Pinçon-Charlot, 1994 ; Caldeira, 1996; Blakely et Snyder, 1997 ; Ghorra-Gobin, 2000 ; Low, 2003; Madoré, 2004 ; Chevalier, Carballo, 2004). Les entretiens effectués ici montrent plusieurs éléments intéressants à ce sujet. En effet, un certain entre-soi est valorisé par les habitants dans le sens où le fait de vivre entre « gens biens » est important. Cela renvoie à la nécessité de se préserver du monde extérieur, de la pauvreté, mais surtout des Pauvres, catalyseurs des peurs et des problèmes de la bourgeoisie recifense. Cet entre-soi est apprécié notamment pour les enfants qui peuvent former des groupes soudés, notamment au sein de l'Escola Internacional de Aldeia, dite l'école américaine, où la quasitotalité des enfants habitant le club sont inscrits. L'homogénéité sociale permet aussi, comme l'espèrent les parents interrogés, de préserver les adolescents d'éventuels problèmes d'alcool ou de drogues. Enfin, le sentiment et le besoin d'ordre, d'organisation, de discipline, passent aussi par cette homogénéité qui garantit, au vu des discours recueillis, un bon ordre social au sein du club (Chevalier, Carballo, 2004).

Mais cet entre-soi affiché s'arrête à ces quelques avantages énoncés. Malgré les fêtes organisées par le club, les différentes activités de loisirs proposées, l'osmose sociale est loin d'exister. Les habitants ont fait état d'un manque de sociabilité, de la difficulté de lier des contacts ou des amitiés. Certaines femmes ont d'ailleurs évoqué la souffrance de leur isolement. Si, à première vue, le Clube de Campo Alvorada peut sembler être un lieu de résidence où la sociabilité, la fraternité et les amitiés caractérisent les rapports humains entre les résidants, au quotidien il n'en est rien pour la plupart des habitants et des usagers du club. À cela, plusieurs raisons. Tout d'abord, les habitants sont majoritairement intégrés à la société du travail. Ils s'absentent donc tous les jours pour travailler, le plus souvent à Recife, hormis les retraités qui représentent quatre personnes 
sur les dix entretiens réalisés. Les retraités en couple ne semblent pas souffrir de cet isolement qui parait être réservé aux femmes veuves ou célibataires qui ne travaillent pas. Sinon, de manière générale, les habitants disent être peu présents au club la semaine et préfèrent se reposer chez eux le week-end. Les modes de vie contemporains, individualistes et urbains, ne favorisent pas une interconnaissance forte du voisinage. Ensuite, l'image d'un lieu où tout le monde se connaît et s'apprécie est fortement idéalisée, étant mise en scène par la structure même du club. Il n'existe pas de communauté affinitaire au sein du club, malgré les caractéristiques particulières du lieu, les modes de sociabilité ne sont pas très différents que ce qui a pu être décrit dans d'autres contextes d'habitat "plus classiques », à savoir une cordiale ignorance. Les principes d'une sociabilité réticulaire, où les habitants connaissent une vie sociale parfois éloignée physiquement de leur lieu d'habitation, ne font pas défaut au Clube de Campo Alvorada (Ascher et Godard, 1999).

Alors même qu'il n'existe pas de communauté affinitaire, les habitants du club ont toutefois des intérêts communs qui justifient leur réunion. En effet, les résidants entretenus disent tous aimer vivre dans une maison, à la campagne, chose qui leur parait impossible de manière individuelle. C'est bien la réunion de plusieurs personnes qui leur permet d'accéder à une protection collective et plus généralement à des services et des équipements auxquels ils ne pourraient pas accéder individuellement, faute de moyens. Cette communauté d'intérêts présente une autre caractéristique intéressante à noter. L'intérêt collectif des habitants se trouve représenté à l'échelle du club. Les revendications, les remarques et les problèmes des habitants sont exprimés à l'échelle du club. Le sentiment collectif se situe au niveau du club qui semble être l'échelle territoriale de référence.

Dans les discours recueillis, le mode de gestion est souvent remis en cause, qualifié de dictatorial, machiste ou plus vaguement «d'un autre temps». Les habitants se plaignent ou regrettent la façon dont est géré le club et surtout de ne pas pouvoir participer au processus décisionnel. Toutefois, aucune réclamation ou action n'a été mise en œuvre. Les quelques tentatives habitantes n'ayant pas eu de conséquences, les résidants disent se résigner à partir du moment où certains services sont garantis. Les habitants considèrent que tant que l'organisation, l'ordre et la propreté règnent dans le club, il ne sert à rien de réclamer, voire de s'engager. Il existe une assez forte déconnection entre les gestionnaires du club et les habitants. Ces derniers ont peu de prises sur le comité directionnel, malgré quelques tentatives, telles que des prises de parole en assemblée ou quelques lettres de réclamations qui sont toujours restées lettres mortes. Malgré cela, le discours sur l'intérêt général ne dépasse pas les frontières de l'espace résidentiel, seule échelle à laquelle les habitants éprouvent un sentiment de maitrise qui, si besoin, deviendrait l'échelle de l'engagement collectif.

\section{Extraits d'entretien}

« La communauté est semblable, tout le monde a le même idéal."

«Et l'école, c'est une grande famille, la majorité des enfants est dans cette école. »

"Je pense que les gens sont comme ça, ceux qui sont de cette époque étaient mieux intégrés. Pas seulement les parents, les enfants aussi. J'ai élevé mes enfants ici, ils ont beaucoup d'amis ici maintenant, ils se retrouvent en bas, ils se respectent beaucoup. C'est génial. Ce sont des gens comme nous, alors il n'y a pas de problèmes de drogue, d'alcool, ils n'ont pas de mauvais comportements comme là dehors. Tous sont formés, de niveau universitaire au moins. »

«Agression, violence et agression. Par exemple, j’adore vivre en maison, mais à Recife, ce n'est pas possible, je ne vais pas vivre en maison, je vais habiter en appartement. Je vais devoir partir d'ici pour un appartement. "

"Une maison isolée, non, je n'aurais pas aimé, c'est dangereux. Une maison isolée, vous devez avoir des vigiles, des gens pour s'en occuper, avoir des équipements. Ici vous avez une équipe de vigiles, des gens qui s'occupent du lieu, alors mon choix fut celui ci. Une maison isolée, je n'aurais pas pu, une copropriété ou un club, mais j'ai trowvé le lieu que je cherchais. » 
"Je voudrais que les choses soient ainsi, plus de réunions, pour les dépenses par exemple, je pense que l'on devrait plus participer.»

"Les décisions arrivent par en haut, c'est très fermé je trouve, rien ne se discute. »

"Je trouve que ce que fait l'administration est anti-démocratique et illégal qui plus est. Mais personne ne veut entrer en justice pour ça.»

«La participation est très faible. »

"Il y a une discrimination envers le sexe féminin. Chaque propriétaire est appelé un membre. Au moment des décisions, vous êtes la femme du membre, parce qu'il y a cette culture où l'homme achète et la femme accompagne, même si dans le couple on partage tout. »

\section{Au fil du temps...}

Il y a parfois un sérieux décalage entre le rêve d'habiter et la réalité, source de déception. Les discours habitants témoignent d'une désillusion qui apparaît au fil du temps.

Les conflits d'intérêts, tout d'abord, sont très nombreux, du fait même de l'existence de deux types d'usagers du club : les habitants permanents et les membres qui n'en profitent que pendant les week-ends et les vacances. Les pratiques et les usages du lieu diffèrent et les plaintes, souvent d'habitants à temps plein deviennent nombreuses quant au « manque de respect » de ceux qui ne viennent que pour profiter et se divertir. De plus, des problèmes d'incivilités, souvent infantiles ou juvéniles sont remarqués, remettant ainsi en cause le calme, la tranquillité et la bonne tenue rêvée. Certains se plaignent du bruit excessif des fêtes, de la conduite dangereuse ou trop rapide de jeunes sans permis dans le club ou encore de petites dégradations des équipements collectifs. Habiter le paradis, les résidants y ont beaucoup fait référence, mais au fur et à mesure du temps et des discours, l'impossibilité de vivre dans un « lieu de vacances » toute l'année se fait ressentir. En effet, la pratique et l'utilisation des équipements de loisirs sont très limitées et le quotidien laisse peu la place à l'allégresse et aux festivités. Comme nous l'avons vu, des conflits se mettent en place et des déceptions émergent.

Au fil du temps, un autre problème surgit, quand les enfants deviennent adolescents. Le paradis de l'enfance peut devenir, avec l'âge, une prison dorée. Les observations ont confirmé le discours habitant à ce sujet. En juillet 2008, vingt-sept titres de club (avec maison) étaient à vendre, dont sept parmi les résidences principales, la principale cause avancée pour justifier la vente étant la déprime adolescente (six annonces sur sept) selon le bureau administratif du club.

Une autre désillusion se fait ressentir, et notamment chez les femmes, vis-à-vis du sentiment d'isolement que certaines peuvent connaître. Les amitiés sont rares, le club est à moitié vide durant la semaine. Elles ont par ailleurs le sentiment d'être peu entendues et encore moins consultées quant aux décisions du club, ce qui accroit leur sentiment de solitude. Les travaux de l'équipe de Guénola Capron montrent que le sentiment d'enfermement et que l'homogénéité sociale a tendance à devenir pesant chez certaines femmes qui oscillent entre l'asphyxie et l'isolement, «elles n'auraient jamais pensé qu'elles pourraient vivre un enfer doré » (p. 166). Dans notre cas, l'isolement, la superficialité des relations et le manque d'amitié véritable est la première source d'insatisfaction.

\section{Extraits d'entretien}

"Vers la fin de semaine, je commence à être tendu, je perds le plaisir et la volonté d'être ici. Il y vient trop de gens, ils arrivent en voiture, avec la musique à fond, ça me stresse vraiment! »

"Au quotidien il y a quelques différends."

"Samedi et dimanche généralement, je lis, je regarde la télé ou un film. Je préfère mienfermer et rester là-haut. Ceux qui viennent le week end, c'est pour boire, s'éclater et ceux qui habitent ici, ce n'est pas leur truc. » 
«Ce que j’aime le moins ce sont les nuits, le week end, cette pagaille, vraiment on doit avoir beaucoup de patience pour supporter. On comprend mais pensez quand à 2 ou 3 heures du matin vous entendez la musique à fond, des rires, des cris de jeunes... c'est l'unique chose qui me déplaise.»

«Les jeunes roulent à toute allure, ils n'ont pas leur permis mais les parents leur prêtent la voiture pour conduire dans le club, avec la musique à fond. Dans ma rue ici, non, mais j'ai déjà entendu des gens réclamer."

"Quand les enfants étaient petits, c'étaient supers, ils aiment beaucoup. Maintenant qu'ils sont adolescents, ils veulent sortir le soir, ils commencent à trouver ça un peu nul. »

"Je n'ai pas beaucoup le temps d'aller à la salle de gym, je fais parfois une petite marche dans le club, le matin ou à la tombée de la nuit.»

"Je ne veux pas forcer, mais j'aimerais avoir des gens plus proches. Je me sens seule. »

"Je ne connais personne, je ne m'engage pas tellement, je n'aime pas les fêtes. "

"C'est comme dans un immeuble, on ne connait pas ces voisins. »

"J'en connais un peu, "bonjour", "bonsoir"."

"Si je veux sortir, discuter ou quelque chose, je n'ai personne sur qui je puisse compter. »

\section{Conclusion}

Le nombre de condominios fechados ne cesse d'augmenter à Aldeia depuis la fin des années 1990. Evidemment, le Clube de Campo Alvorada est un des plus anciens, les modalités de pratiques et de gestion sont celles d'un club et tout ceci le rend à divers égards différent des copropriétés fermées qui se sont construites plus récemment. Mais le modèle urbain est similaire : des populations aisées viennent se mettre au vert et au frais à quelques kilomètres de Recife, au sein d'ensembles résidentiels fermés et sécurisés où divers services et équipements leurs sont proposés.

Les représentations habitantes analysées ici corroborent avec les premières recherches menées notamment par l'équipe de G. Capron sur les countries clubs de l'arrière pays de Buenos Aires. L'idéalisation d'un lieu voulu et rêvé comme parfait, l'entre-soi qui garantit une bonne tenue, mais qui permet surtout d'accéder à des prestations collectives de qualité, sont les caractéristiques prédominantes de ce mode d'habiter. En revanche, la bonne sociabilité ou la communauté affinitaire est loin d'être vérifiée malgré des relations de voisinages cordiales. Enfin, des déceptions et des désillusions peuvent apparaître et noircir le tableau idyllique d'un lieu paradisiaque.

En quoi ces figures dévoilées par l'analyse de la parole habitante renseignent-elles sur la manière d'habiter les villes contemporaines? Les inégalités socio-spatiales ne sont pas récentes au Brésil, elles sont même parfois considérées comme la base de la structure nationale. En revanche, la nouveauté est l'importance donnée aux loisirs et au plaisir, à la détente et au farniente, à l'hédonisme en somme comme mode de vie (Leal, 2008). Même si les équipements de loisirs sont largement sous-utilisés au quotidien et que l'idée des vacances toute l'année est difficile à concilier avec les prérogatives du travail, l'image des loisirs et du plaisir au jour le jour reste très attractive. La surenchère sécuritaire semble être aussi une caractéristique contemporaine. Les espaces résidentiels sont de plus en plus imperméables et la tendance amène à toujours plus d'équipements de sécurisation sans qu'il ait été fait état d'actes d'intrusion ou de violence. Les effets de mode ou de mimétisme imprègnent les prises de décisions au sein des ensembles résidentiels.

La municipalité de Camaragibe s'inquiète du risque d'une trop grande fragmentation de son territoire en laissant s'installer ces enclaves de populations fortunées sur le plateau d'Aldeia, quand le bas de la ville reste majoritairement très pauvre. Malgré tout, ces populations représentent un apport de recette fiscale et une valorisation de la commune que la mairie ne voudrait pas voir disparaitre. Aussi, la majeure partie d'Aldeia est classée en zone de protection environnementale. Au dernier Plan Directeur (équivalent du Plan Local d'Urbanisme), achevé en 2008, le parti pris avait été de limiter la densité possible de constructions. La municipalité se sert ainsi de l'essor de ces enclaves pour protéger les ressources naturelles. Privatiser pour mieux protéger, tel est le 
concept clé en termes de politique environnementale, avec le risque croissant d'une ségrégation accentuée et d'un droit à la nature, à un espace de qualité de plus en plus compromis pour une grande partie de la population.

\section{Bibliographie}

Ascher (F.) et Godard (F.), 1999. - «Vers une troisième solidarité », Esprit, n 11, p. 168-189.

Billard (G.), Chevalier (J.) et Madoré (F.), 2005. - Ville fermée, ville surveillée. La sécurisation des espaces résidentiels en France et en Amérique du Nord, édition Presses Universitaires de Rennes, coll. "Géographie Sociale », 220 p.

Blakely (E. J.) et Snyder (M. G.), 1997. - Fortress of America: Gated communities in the United States, Cambridge, Washington DC, Brooking Institution Press, Lincoln Institute of Land Policy.

Capron (G.) (dir.), 2006. - Quand la ville se ferme. Quartiers résidentiels sécurisés, Paris, Bréal, coll. « D’Autre Part », 288 p.

Caldeira (T.), 2003. - Cidade de muros : crime, segregação e cidadania em São Paulo, São Paulo, Seconde édition (première édition 1996), Ed. 34/Edusp, 400 p.

Chevalier (J.) et Carballo (C.), 2004. - «Fermetures résidentielles et quête de l'entre-soi, entre Nord et Sud des Amériques », L'Espace géographique, n 4, p. 325-335.

Degoutin (S.), 2006. - Prisonniers volontaires du rêve américain, Paris, édition de la Villette, 396 p.

Freyre (G.), 1933. - Casa Grande E Senzala : formação da família brasileira sob o regime de economia patriarcal. Rio de Janeiro, Mais \& Schmiat.

Freyre (G.), 2004. - Sobrados e Mocambos : decadência do patriarcado rural e desenvolvimento urbano, São Paulo, 15édition (Première édition en 1936), Global Editora, 968 p.

Ghorra-Gobin (C.), 2000. - «États-Unis : gated communities et private cities », Urbanisme, n³12, p. 67-69.

Glasze (G.), Webster (C.), Frantz (K.) (dir.), 2005. - Private Cities. Global and Local Perspectives, Londres, Routledge, $242 \mathrm{p}$.

Lacarrieu (M.), Thuillier (G.), 2004. - « Une utopie de l'ordre et de la fermeture : « quartiers privés » et « countries » à Buenos Aires », L’Espace géographique, n² 2, p. 149-164.

Leal (S.) (coord.), 2008. - Pesquisa Arranjos Institucionais de Governança e Produção Imobiliária na Metrópole do Recife, Recife, Relatório técnico-científico, Núcleo de Gestão Urbana e Políticas Públicas, 137 p.

Le Guirriec (P.), 2008. - «Condominios horizontais e tipos de sociabilidade », Fortaleza, Simpósio Espaço Metropolitano, Turismo e Mercado Imobiliário, Observatório das Metrópoles, Núcleo Fortaleza, Mestrado em geografia da UFC, 18 juin, 12 p.

Leitão (L.), 2005. - «Quando um muro separa e nenhum ponte une », Cadernos Metrópoles, $n^{\circ} 13,1^{\text {er }}$ semestre, p. 229-253.

Low (S.), 2003. - « Construire l'exclusion à travers les communautés fermées ", Les Annales de la Recherche Urbaine, $\mathrm{n}^{\circ}$ 93, p. 149-157.

Madoré (F.), 2004. - «Géographie et modalités de la fermeture des espaces résidentiels en France », L'information géographique, $\mathrm{n}^{\circ} 2$, p. 155-172.

Pinçon (M.) et Pinçon-Charlot (M.), 1994. - «Propriété individuelle et gestion collective », Les Annales de la Recherche Urbaine, ${ }^{\circ}$ 65, p. 35-46.

Thuillier (G.), 2001. - « Les quartiers enclos à Buenos Aires : quand la ville devient country», Cahiers des Amériques latines, $\mathrm{n}^{\circ} 35$, p. 41-56.

Vuaillat (F.), Madoré (F.), 2008. - « La parole habitante au sein d'une résidence fermée : le Newton à Carquefou », Cahiers Nantais, n 1, p. 41-50.

Cet article a été reçu le 6 février 2009 et définitivement accepté le 6 août 2009. 